\title{
Overload Control for Massive Machine Type Communications
}

\author{
Ray-Guang Cheng \\ Professor \\ Department of Electronic and Computer Engineering, \\ National Taiwan University of Science and Technology, \\ Taipei, Taiwan, R.O.C. \\ crg@mail.ntust.edu.tw
}

Massive machine type communication (MTC) is one of the use cases for IMT-2020. Concurrent accesses of a cellular network by mass MTC devices may result in intolerable delays, packet loss, or even service unavailability to human-tohuman $(\mathrm{H} 2 \mathrm{H})$ communication services. Hence, proper overload control mechanisms are required to guarantee network availability and quality of $\mathrm{H} 2 \mathrm{H}$ services under heavy MTC load. In this lecture, we introduce the current 3GPP Long Term Evolution Advanced (LTE-A) random access procedure and the radio access network overload control solutions within LTE/LTE-A. An overview of the several considered overload control solutions, including the standardized Extended Access Barring (EAB) solution, will be briefly introduced. We present an iterative contending-user estimation (ICE) method for the analysis of multi-channel slotted ALOHA protocol in transient conditions and with non-stationary arrivals. We then demonstrate how to use the ICE method to model LTE-A random access procedure and estimate the RACH performance under different overload control schemes. 